\title{
Micro-CT based structure tensor analysis of fibre orientation in random fibre composites versus high-fidelity fibre identification methods
}

\author{
Radmir Karamova , Luca M. Martulli ${ }^{\text {b,c }}$, Martin Kerschbaum ${ }^{c}$, Ivan Sergeichev ${ }^{a}$, Yentl \\ Swolfs ${ }^{b}$ and Stepan V. Lomov ${ }^{b *}$ \\ ${ }^{a}$ The Centre for Design, Manufacturing and Materials, Skolkovo Institute of Science and \\ Technology, Bolshoy Boulevard 30, bld. 1 Moscow, Russia 121205 \\ b Department of Materials Engineering, KU Leuven Kasteelpark Arenberg 44, 3001, \\ Leuven, Belgium \\ ${ }^{c}$ Toyota Motor Europe, Material Engineering, Hoge Wei 33 - 1930 Zaventem, Belgium \\ *Corresponding Author: stepan.lomov@kuleuven.be; +32-16-32-12-10
}

\begin{abstract}
The fibre orientation distribution controls the mechanical properties of random fibre composites. Generally accepted methods for its characterisation involve identification of fibres or their ellipsoidal cross sections as individual objects, requiring high image resolution and high computational resources. This paper investigates whether structure tensor analysis can be an alternative and whether it can work with lower resolution images. Micro-computed X-ray tomography images of random glass fibre/polypropylene injection moulded composites were processed using ellipsometry on 2D slices, 3D fibre identification (Avizo software) and analysis of the structure tensor (VoxTex software). The images had resolutions of 1.4, 3.2, 8 and $16 \mu \mathrm{m}$ per pixel, compared to an average glass fibre diameter of $17 \mu \mathrm{m}$. All the methods yielded similar results for high-resolution images $(1.4$ and $3.2 \mu \mathrm{m})$. The high-fidelity, direct identification of fibres failed for low-resolution images, but the structure tensor analysis still yielded results close to the high-resolution scans.
\end{abstract}

Keywords: random fibre composites; micro-computed tomography; micro-structural analysis; orientation tensor; orientation distribution

\section{Introduction}

Random short fibre composites become increasingly effective in various industries, particularly in automotive [1]. One of the main characteristics of short fibre composites is the Fibre Orientation Distribution (FOD), which determines the microstructure and therefore the properties of the entire material. FOD is most often characterised by orientation tensors [2]. FOD of injection moulded random fibre composites can be predicted with so-called "reinforced flow" methods, as illustrated in the pioneering work of Bay and Tucker [3] and reviewed by Binetruy et al. [4]. The mechanical behaviour can therefore also be calculated. Experimental measurements of the FOD, however, remain challenging.

In one of the most frequently used methods, the elliptic shapes of fibre cross sections are measured on two-dimensional (2D) microscopy images of polished specimens [5-9]. These ellipsometrybased methods have limitations due to (1) the ambiguity of resolving the fibre orientation based on the elliptical cross-section and (2) loss of precision for fibres close to normal or parallel to the cross-section plane. These measurements are often also impractical: a large amount of data must be processed, and the identification of the individual cross sections often requires operator intervention.

In the last decade, important breakthroughs have been made in analysis of FOD based on identifying fibres as three-dimensional objects in micro-Computed Tomography (micro-CT) 
scans [10-13]. Some are implemented in commercial software (for example, Avizo, GeoDict, VGStudio Max), which can be used for detailed characterisation of fibre orientation $[14,15]$. However, these methods require high-resolution scans, high computational resources and analysis time, and face difficulties in segmentation of closely spaced fibres. In the present paper, we will label them as "high-fidelity" methods. Avizo was used in the present work, as a representative example of implementations of the high-fidelity methods based on identification of individual fibres.

Fibre orientation can also be evaluated through global measurements when the orientation of each individual fibre is not known. One of the first and still widely used global measurement methods is mean-intercept length (MIL) [16-18], which was later extended to the volume orientation (VO) method [19]. MIL and VO methods were compared with Avizo's high-fidelity fibre tracing method XFIBER by De Pascalis and Nacucchi [12]. They compared three materials, including short fibre composites, but only for high-resolution micro-CT scans. They found a good correspondence between MIL/VO and the high-fidelity Avizo method. Other studies have established relationships between image resolution and errors in fibre orientation calculations $[10,20]$. The low-resolution scans in these studies were achieved by synthetic datasets or by Lanczos resampling of high-resolution scans of real material. However, the real data acquired by low-resolution micro-CT may differ from synthetically generated data.

Straumit et al. [21] proposed a method to evaluate the fibre orientation distribution of fibre reinforced composites based the micro-CT scans analysis using local structure tensors of the image. The method is implemented in the VoxTex software and was used for characterisation of the fibre orientation in different fibre-reinforced composites and for transformation of micro-CT 3D images into finite element models [22-26].

The structure tensor analysis is an indirect method, and it should be validated by comparison with high-fidelity methods. Such validation was done in [21] for unidirectional long fibre composites with the main validation parameter being the misalignment of the fibres but has not been performed yet for random short fibre composites, for which full FOD should be considered.

This paper investigates the reliability of the structure tensor analysis of fibre orientation in short fibre composite in comparison with high-fidelity methods. FOD measurements of random short glass fibre composites are performed using micro-CT images of different resolution with various methods: classical ellipsometry on the fibres seen on high-resolution micro-CT slices, 3D fibres reconstruction and subsequent orientation identification (Avizo software) and structure tensor analysis (VoxTex software).

\section{Identification of the fibre orientation distribution and the orientation tensor}

\subsection{FOD, orientation tensors and their comparison metrics}

The orientation of a single straight fibre can be described by the spherical polar $\varphi$ and $\theta$ angles of its centre-line (see Figure 1a). Fibre orientation distribution function (ODF) $\psi(\varphi ; \theta)$ gives the most general description of fibres orientation state in space:

$P\left(\theta_{1} \leq \theta \leq \theta_{1}+d \theta, \varphi_{1} \leq \varphi \leq \varphi_{1}+d \varphi\right)=\psi\left(\theta_{1}, \varphi_{1}\right) \sin \theta_{1} d \varphi d \theta$,

where $P(\ldots)$ is the probability of finding the orientation given in brackets. The ODF can also be expressed as $\psi(\boldsymbol{p})$, where $\boldsymbol{p}$ is a unit vector $\boldsymbol{p}=\left(p_{1}, p_{2}, p_{3}\right)=(\sin \theta \cos \varphi, \sin \theta \sin \varphi, \cos \theta)$. 


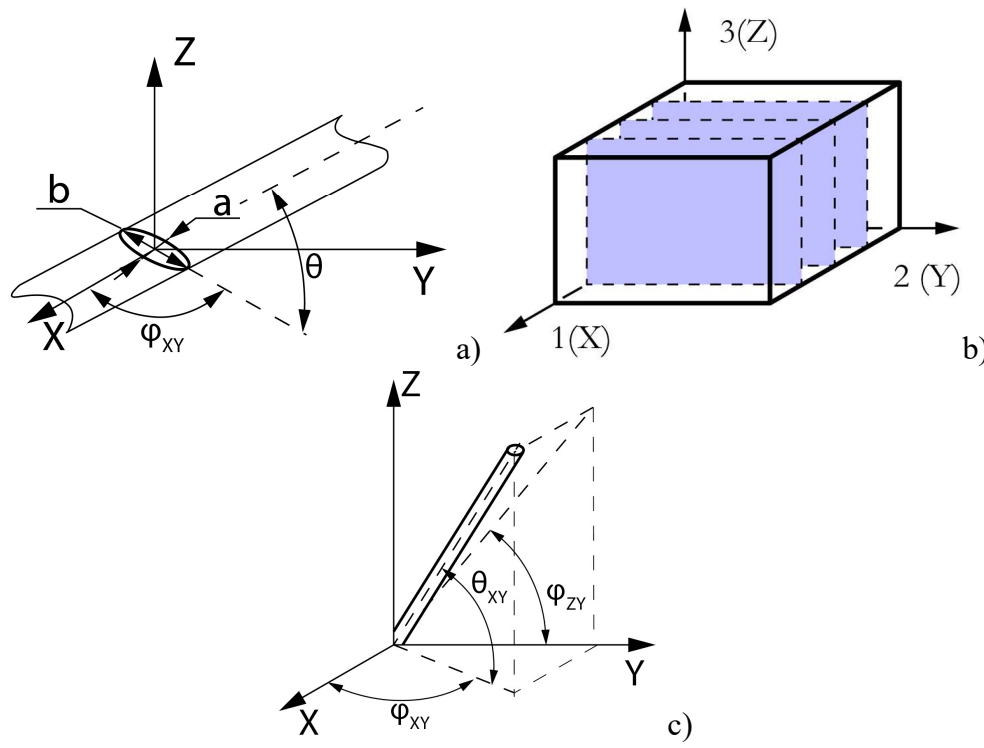

Figure 1: Ellipsometry: (a) orientation angles of a single fibre and elliptical section of the fibre; (b) cross sections of micro-CT scans used for ellipsometry: main slices orthogonal to X axis are shown; (c) $\theta_{X Y}$ angle can be reconstructed from $\varphi_{X Y}$ and $\varphi_{Z Y}$.

Despite the fact that ODFs provides a full description of the fibre orientation state, it is difficult to use them in an efficient way. Orientation tensors are a concise description of FOD [2]. The general form of the second-order orientation tensor is

$a_{i j}=\oint p_{i} p_{j} \psi(\boldsymbol{p}) d \boldsymbol{p}$

or, in a discretized form when an assembly of individual fibres $\left\{\boldsymbol{p}^{k}\right\}_{k=1}^{n}$ is considered:

$$
a_{i j}=\frac{1}{n} \sum_{k=1}^{n} a_{i j}^{k}=\frac{1}{n}\left(\sum_{k=1}^{n} p_{i}^{k} p_{j}^{k}\right)
$$

Two orientation tensors can be compared using such measures as degree of orientation, cosine similarity or using tensor difference measure for all or trace components [27,28]. The degree of orientation $O_{D}$ is a scalar value, which expresses the difference between the FOD in question and the isotropic orientation distribution. This value can be calculated by normalizing the largest eigenvalue $\lambda_{1}^{O T}$ of the orientation tensor:

$$
O_{D}=\frac{3}{2}\left(\lambda_{1}^{O T}-\frac{1}{3}\right)
$$

The cosine similarity value compares the main direction of the fibre orientations. The cosine similarity is calculated using dot product of the vectors $\left(\boldsymbol{V}_{1}\right.$ and $\left.\boldsymbol{V}_{2}\right)$ which represent the main directions of the investigated OFDs or using the angle $\alpha$ between these two directions can be calculated:

$$
\text { Similarity }_{C}=\left|\boldsymbol{V}_{1} \times \boldsymbol{V}_{2}\right|=|\cos \alpha|
$$

If the whole information of orientation tensor is compared, the tensor difference measure can be used: 
Difference $_{T}=\sqrt{\sum_{i=1}^{3} \sum_{j=1}^{3}\left(a_{i j}-b_{i j}\right)^{2}}$

\subsection{Direct methods for FOD measurement}

Two high-fidelity direct methods for FOD measurement will be used: ellipsometry and 3D fibre reconstruction.

\subsubsection{Ellipsometry}

$\varphi$ and $\theta$ angles of a single fibre can be retrieved based on the fibre ellipsoidal cross sections (see Figure 1a). To this end, 2D microscope images or slices of micro-CT scans of short fibre composite materials can be used.

$\varphi$ angle of the fibre corresponds to the direction of the major ellipse axis, which can be found directly from the image. $\theta$ angle is calculated based on the ellipse axes length:

$|\cos (\theta)|=\frac{b}{a}$

where $b, a$ - the ellipse minor and major axis respectively. Equation (7) introduces ambiguity in measurements because it has two solutions: $\arccos \left(\frac{b}{a}\right)$ and $\pi-\arccos \left(\frac{b}{a}\right)$. If micro-CT slices are used, this ambiguity can be resolved by using an additional slice at 3-5 $\mu \mathrm{m}$ distance from the main one (Figure 1b). A simple algorithm was developed to find the same fibre in two neighbourhood slices using the cross section of the fibre from the first slice as search area of the centre in the second slice. This algorithm allows making a choice between the two solutions and completely removes the ambiguity.

Fibres oriented close to perpendicular or parallel to the cutting plane cause error in calculations of $\theta$ angles, which are close to $0^{\circ}$ and $90^{\circ}$ in these cases. The errors appear due to large sensitivity of the solution of (7) to errors in identification of the major and minor axes of an ellipse. The errors can be decreased by using three mutually perpendicular section planes XY, ZX, YZ (see Figure 1b), which is possible with micro-CT scans, but difficult with physical cross-sectioning.

From $X Y$ plane, $\varphi_{X Y}$ angle is identified and $\varphi_{Z X}, \varphi_{Y Z}$ projection angles are determined from two other (ZX and YZ) planes (Figure 1c). This information is sufficient to obtain the reconstructed FOD, because it is possible to reconstruct $\theta_{X Y}$ angle from one of the projection angles and $\varphi_{X Y}$ :

$\theta_{X Y}=\arctan \left(\sin \varphi_{X Y} \tan \varphi_{Y Z}\right)$

Then mainly the $\varphi_{X Y}$ and the $\varphi_{Z X}, \varphi_{Y Z}$ projected angles will be considered in this method since they have less calculation errors.

A single fibre with length $\mathrm{L}$ and diameter $\mathrm{D}$ can appear in a randomly placed cross-section with the probability that is proportional to the projected length of the fibre on the z-axis [29]:

$P(\theta)=(L \cos \theta+D \sin \theta) K$

where $\mathrm{K}$ is a constant, $L$ and $D$ are the fibre length and diameter. To account for errors on ODF associated with this probability, Zhu et al. [29] introduced a weighting function $g(\theta)$ :

$g(\theta)=\frac{p(\theta=0)}{p(\theta)}=\frac{r}{r \cos \theta+\sin \theta}$ 
where $r=L / D$ is the fibre aspect ratio. Therefore, when the orientation tensor is calculated, contribution of each fibre orientation must be weighted:

$$
a_{i j}=\frac{\sum_{n=1}^{n} g_{n}\left(a_{i j}\right)_{n}}{\sum_{n=1}^{n} g_{n}}=\frac{\sum_{n=1}^{n} \frac{1}{L \cos \theta_{n}+D \sin \theta_{n}}\left(p_{i} p_{j}\right)_{n}}{\sum_{n=1}^{n} \frac{1}{L \cos \theta_{n}+D \sin \theta_{n}}}
$$

If the aspect ratio of the fibres is large $(L>>D)$,

$$
a_{i j}=\frac{\sum_{n=1}^{n} F_{n}\left(a_{i j}\right)_{n}}{\sum_{n=1}^{n} F_{n}} \text {. }
$$

where the $F_{n}$ describes the weighting function for the n-th fibre [8]:

$$
\left.\begin{array}{ll}
F(\theta, L, D)=1 /(L \cos \theta) & \text { for } \theta<\theta_{\text {krit }} \\
F(\theta, L, D)=1 / D & \text { for } \theta_{\text {krit }}<\theta<90
\end{array}\right\} \text {, where } \theta_{\text {krit }}=\arccos \frac{D}{L}
$$

\subsubsection{D fibre assembly reconstruction}

A single fibre can be reconstructed by a fibre-matrix segmentation of each micro-CT slice and tracing the fibre on these slices through the scan [30]. This method is computationally very expensive. Another method involves the local normalized cross-correlation with a template of a cylinder and then tracing the centreline of the templates to reconstruct a fibre [31,32]. This method is implemented in Avizo software, and the reader is referred to its documentation [33] for a detailed explanation.

When all the fibres in the assembly are reconstructed, the histograms of the ODF are built, and the orientation tensor is calculated using (3).

\subsection{Structure tensor}

Structure tensor analysis is a widely used technique in image processing, including processing of micro-CT images [34-37]. For fibre-reinforced composite materials, this technique was implemented in the VoxTex software by Straumit et al. [21]. In a micro-CT scan represented as a three-dimensional grey value function of coordinates $I\left(x_{1}, x_{2}, x_{3}\right)$, microstructural anisotropy is indicated by the grey value gradient. From this gradient, the principal direction of anisotropy can be calculated using a structure tensor. The continuous version of the structure tensor for threedimensional space is defined as follows:

$$
S(\boldsymbol{q})=\int_{W(\boldsymbol{q})} S^{\prime}(\boldsymbol{r}) d r, S^{\prime}\left(x_{1}, x_{2}, x_{3}\right)=\left[\begin{array}{lll}
\left(\frac{\partial I}{\partial x_{1}}\right)^{2} & \frac{\partial I}{\partial x_{1}} \frac{\partial I}{\partial x_{2}} & \frac{\partial I}{\partial x_{1}} \frac{\partial I}{\partial x_{3}} \\
& \left(\frac{\partial I}{\partial x_{2}}\right)^{2} & \frac{\partial I}{\partial x_{2}} \frac{\partial I}{\partial x_{3}} \\
& \left(\frac{\partial I}{\partial x_{3}}\right)^{2}
\end{array}\right] ;
$$

where vector $\boldsymbol{q}$ represents the centre of an integration window $W(\boldsymbol{q})$. The volume of the integration window is defined by a discrete parameter $w_{r}$ that is referred further as window size. The actual volume of the integration window is $\left(2 w_{r}+1\right)^{3}$ pixels. The distance between window centres can be smaller, equal or larger to the window size. The values of the partial derivatives 
sampled at point $\boldsymbol{r}\left(x_{1}, x_{2}, x_{3}\right)$ may be estimated by finite difference formulae. Straumit et al. [21] proposed to calculate the derivatives with a 5-point numerical differentiation scheme.

The eigenvalues of the structure tensor $\lambda_{1}, \lambda_{2}, \lambda_{3}$ and the corresponding eigenvectors $\boldsymbol{v}_{\mathbf{1}}, \boldsymbol{v}_{\mathbf{2}}, \boldsymbol{v}_{\mathbf{3}}$ describe the distribution and the variability of the gradient directions within the integrated window. The eigenvector with the smallest eigenvalue indicates in which direction the microstructure is least variable. This direction is the principal direction of anisotropy within this window and it corresponds to the local fibre direction.

With the knowledge of the principal direction of anisotropy in each voxel, the calculations of the ODF for these directions is straightforward. Normalisation of $\theta$ in $\left(0,180^{\circ}\right)$ and $\varphi$ in $\left(-90^{\circ}, 90^{\circ}\right)$ is used to avoid ambiguity. The ODF of the voxel orientations is taken as the ODF of the fibres in the volume. The orientation tensor is then calculated with equation (3), using $3.6^{\circ}\left(=180^{\circ} / 50\right)$ discretisation of the ODF histogram in $\varphi$ and $\theta$ angles. In addition, the degree of anisotropy can be determined as:

$$
\beta=\left\{\begin{array}{c}
1-\frac{\lambda_{1}}{\lambda_{3}}, \quad \lambda_{3}>0 \\
0, \quad \lambda_{3}=0
\end{array},\right.
$$

Isotropic materials such as air or matrix have a degree of anisotropy close to 0 , whereas the degree of anisotropy of a unidirectional fibre bundle tends to 1 .

\section{Materials and methods}

\subsection{Materials}

The material used in the present work is a random glass fibre polypropylene composite. The specimens were provided by Toyota Motor Europe. The specimen was cut from a flat injection moulded component. The average fibre diameter is $17 \mu \mathrm{m}$, the average fibre length was $719 \mu \mathrm{m}$ and the median was $643 \mu \mathrm{m}$. The glass fibre weight content was $40 \%$. 3.2 Micro-computed tomography

A GE Phoenix Nanotom CT system was employed. The system is equipped with a $180 \mathrm{kV} / 15 \mathrm{~W}$ nanofocus X-ray tube and a $2300 \times 2300$ pixel on a 12-bit Hamamatsu flat panel detector.

The specimen for micro-CT imaging was cut in the shape of a rectangular cuboid of dimensions $2 \mathrm{~mm} \times 2 \mathrm{~mm} \times 20 \mathrm{~mm}$.

The same volume was scanned with four resolutions: for $1.4,3.2,8$ and $16 \mu \mathrm{m}$ per pixel. The corresponding images will be called below for brevity " $1.4 \mu \mathrm{m}$ image" and so on. For the $1.4 \mu \mathrm{m}$ images, three locations were scanned to obtain images of the whole specimen, and two locations for $3.2 \mu \mathrm{m}$ images.

The accelerating voltage and the beam current of the X-ray tube were $60 / 90 \mathrm{kV}$ and $220 / 300 \mu \mathrm{A}$, respectively, for $1.4 / 3,2 \mu \mathrm{m}$ image. $8 \mu \mathrm{m}$ and $16 \mu \mathrm{m}$ resolutions were obtained with the same parameters as $3.2 \mu \mathrm{m}$ image by changing the distance between the target and the specimen. In all cases, the detector integration time was $0.800 \mathrm{~s}$ and the number of projections was 2400 . A molybdenum target was used, as this was most suitable for weak absorbing materials.

\subsection{Data processing}

Figure 2a shows an example of a micro-CT scan. The micro-CT scans revealed that a skin-core effect was present (see Figure $2 b$ ). It was decided to analyse only the core part of the composite, which was called the volume of interest (VOI) in Figure 2a and b. The VOI has the same dimensions for the images of all resolutions: $2611^{*} 2436^{*} 655 \mu \mathrm{m}^{3}$. To analyse the same VOI on the images of different resolutions, a specific feature in the form of three broken fibres was found 
on $1.4 \mu \mathrm{m}$ and $3.2 \mu \mathrm{m}$ images, and knowing its location, the same VOI were analysed for the other resolutions. The result of the data processing is fibre orientation distributions in the VOI.
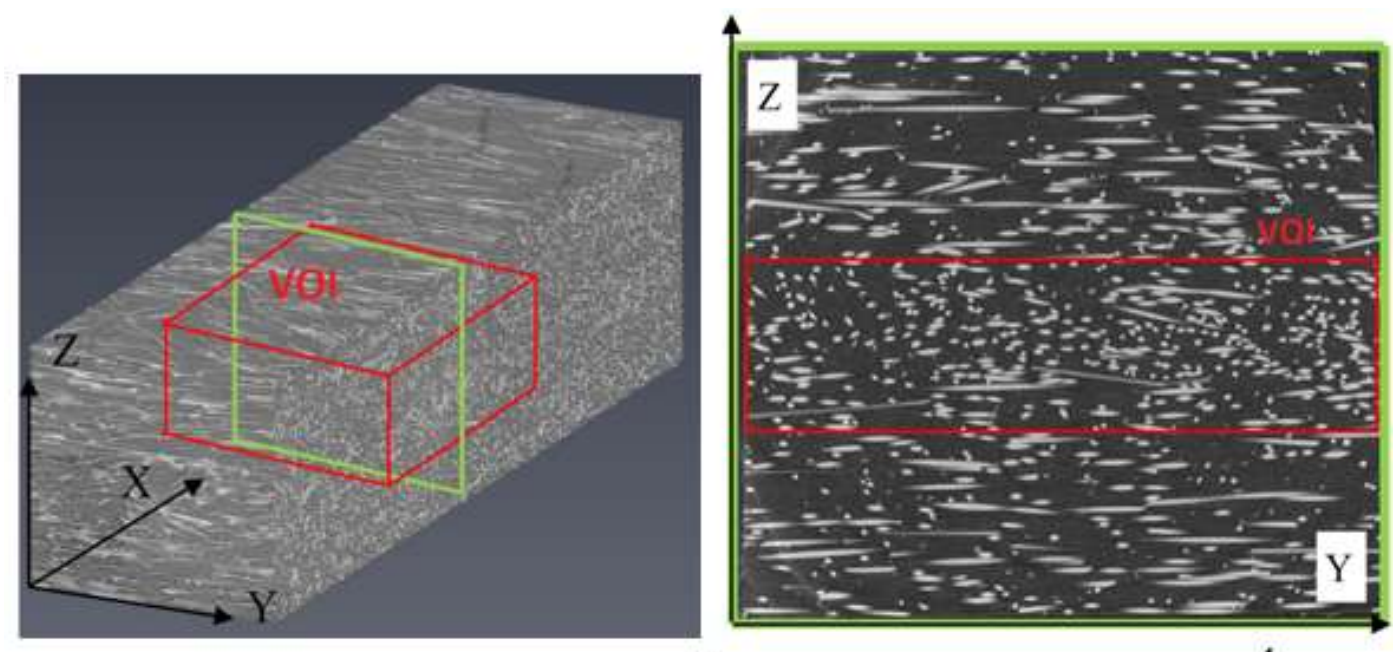

a

b

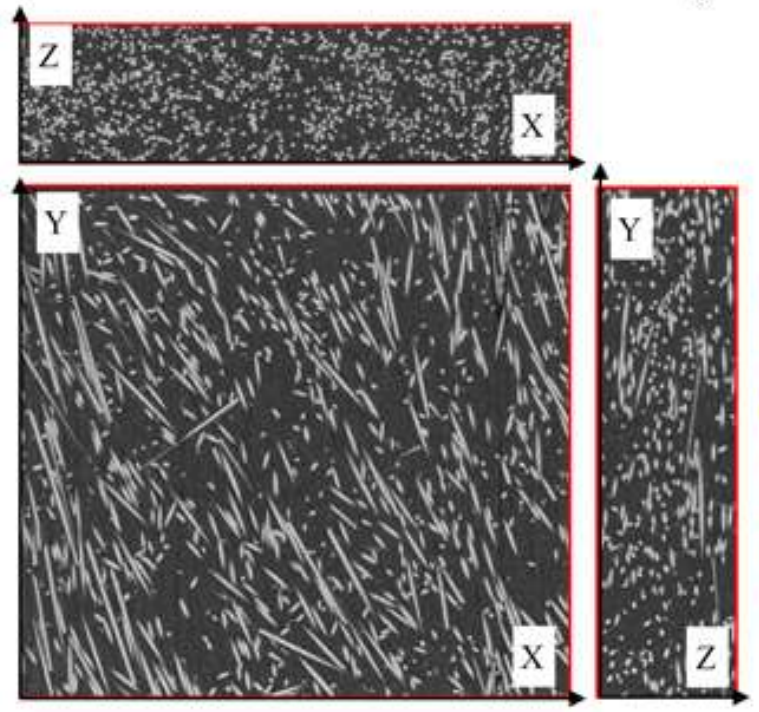

c

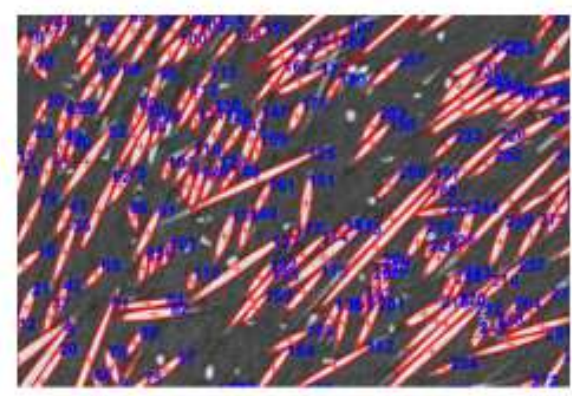

d

Figure 2: Micro-CT images, resolution $1.4 \mu \mathrm{m} /$ pixel: (a) $3 D$ reconstruction of the specimen with VOI (red) and ZY plane (green) (b) the core-skin effect and the VOI projection on the plane ZY (c) VOI projections on $X Y, Z Y, Z X$ planes, and (d) a typical image processed in Matlab with the Trainable Weka Segmentation plugin. VOI size in (a-c) is $1865 * 1740 * 475$ pixels or $2611 * 2436 * 665 \mu \mathrm{m}$.

Following the approach of [10,20], the $1.4 \mu \mathrm{m}$ images were used to obtain synthetically resampled $3.2 \mu \mathrm{m} /$ pixel resolution images using bilinear interpolation (implemented in ImageJ software). The new resampled $3.2 \mu \mathrm{m} /$ pixel resolution images further are referred as $3.2 \mu \mathrm{m}$ downscaled images, and will be used to evaluate sensitivity of high-fidelity methods to the image resolution.

\subsubsection{Ellipsometry}

The VOI sections for ellipsometry were defined as follows. In each of the three slicing directions, three main slices were selected that divide the VOI into four equal parts (see Figure 2c). For each main slice, an additional slice is made at the distance of 4 pixels from the main slice. The 
additional slices were used to solve the ambiguity in the calculation of $\theta$ angles. In total, there are 18 slices per VOI, which are analysed for each of the four resolutions.

Image J (Fiji) and Matlab image processing tools were used to measure elliptical parameters of the fibre cross-sections. To segment closely spaced fibres the Trainable Weka Segmentation plugin of ImageJ [38] was used. Ellipses were detected using the regionprops function of Matlab, and they are then filtered by area and minor axis to remove chunks and falsely detected objects from the calculations. The final image with the identified ellipses is shown in Figure 2d, showing excellent fibre recognition. OFD histograms, orientation tensors, mean values and standard deviations are calculated using built-in Matlab functions for the set of ellipses reconstructed on each of the slices.

\subsubsection{Avizo}

Avizo requires high resolution micro-CT so that it is possible to distinguish each fibre. Therefore, $1.4 \mu \mathrm{m}$ images were used for 3D identification of individual fibres. The fibre tracing procedure starts with the computation of the correlation between a cylindrical template and the cylinder correlation module. This module requires a CUDA (Compute Unified Device Architecture) computational device to run and a considerable computational time: for a $1865 \times 1740 \times 475$ image $(1.4 \mu \mathrm{m}$ VOI) it took about 22 hours on HP Z800 workstation (two CPUs $2.67 \mathrm{GHz}$ : two processors and 12 cores, RAM 98 GB, GPU Nvidia Geforce GTX970: 4 GB GDDR5, 13 processors and 104 CUDA cores). The following parameters were used: a cylinder length of 300 pixels, an angular sampling of 5, a mask cylinder radius of 15 pixels, and an outer cylinder radius of 8 pixels. After that, the centre-line tracing stage was performed using master correlation with a minimum seed correlation of 100 . A search cone with an aperture angle of $37^{\circ}$ and the direction coefficient of 0.1 were used. The final step was to convert the trace geometry to labels using watershed algorithms and then to extract statistical data. Due to the long calculation time, the calculations were made only with respect to one coordinate system.

\subsubsection{Structure tensor}

In the present work, VoxTex software was used, employing the structure tensor analysis. The micro-CT scans are uploaded to the program, and a voxel model is created. A voxel contains $n^{3}$ pixels of the initial micro-CT scan. The value $n$ was chosen in such a way that the voxel size is close to the glass fibre diameter. The size of the integration window (see equation (8)) was chosen as $w_{r}=16$ for $1.4 \mu \mathrm{m}$ images, 10 for $3.2 \mu \mathrm{m}$ images, 5 for $8 \mu \mathrm{m}$ images and 3 for $16 \mu \mathrm{m}$ images. Each voxel of the model has information about the average grey scale (represents material density), degree of anisotropy and its principal direction. Figure 3a represents a densityanisotropy 2D histogram:- the y-axis plots the degree of anisotropy, which reveals clusters corresponding to the fibres (high anisotropy, high density) and matrix (low anisotropy, low density). An anisotropy histogram of a voxel model has two peaks (see Figure 3b,c). The peak with small degree of anisotropy represents the matrix, whereas the one with higher values represents the fibres. In the subsequent analysis the filter is used so that only the voxels with a high anisotropy value are analysed, excluding full-matrix voxels. In each case, the threshold is chosen manually. 


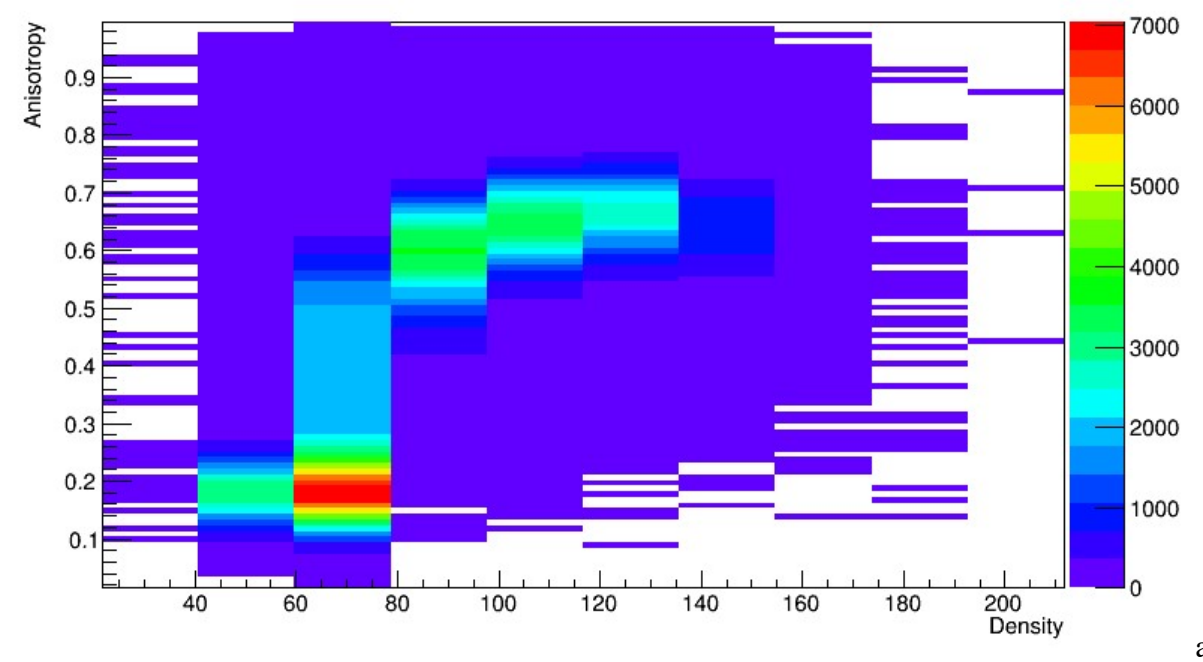

a)
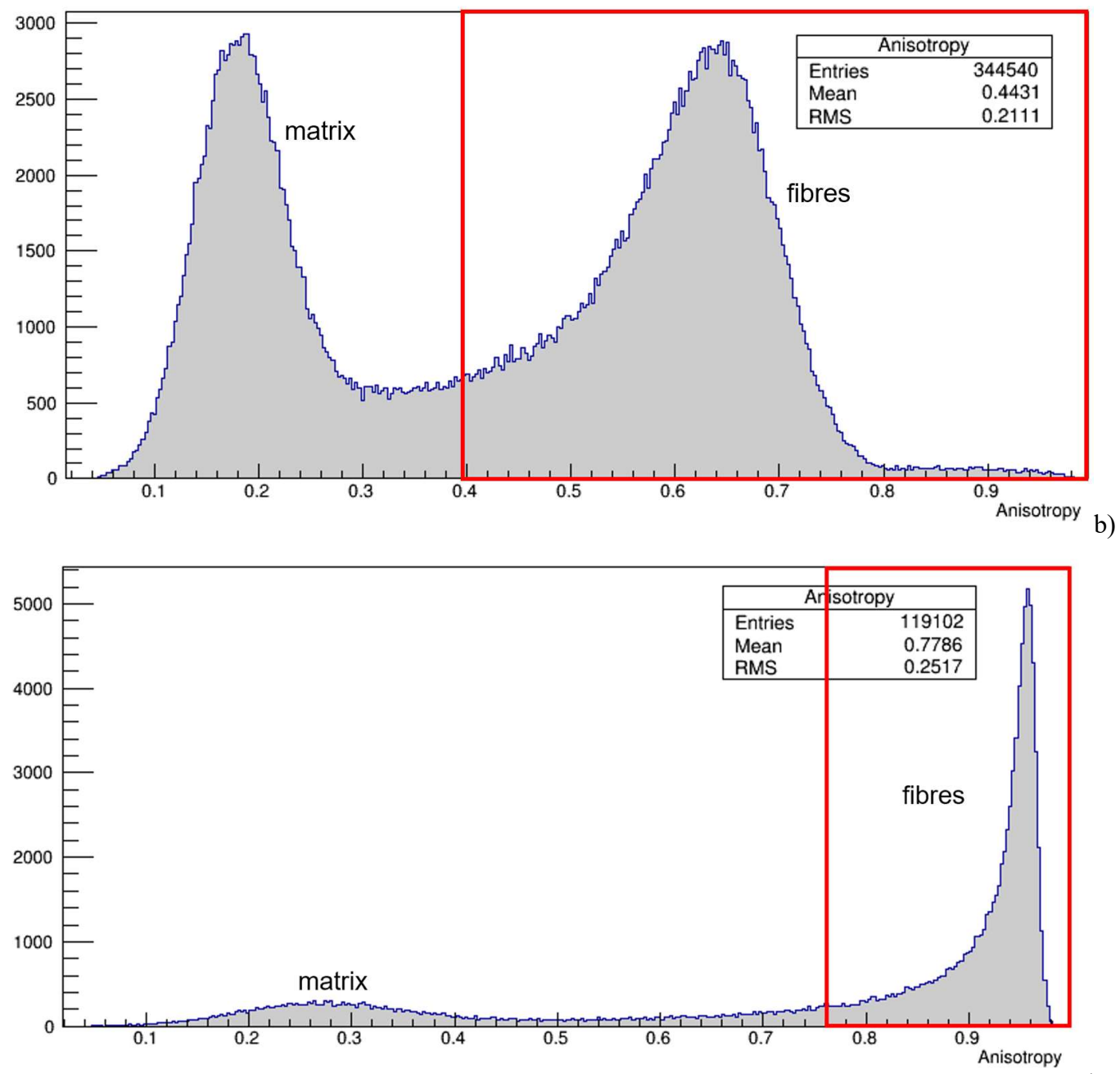

c)

Figure 3: Definition of the anisotropy threshold: (a) 2D density-anisotropy histogram for $1.4 \mu m$ scans, (b) the threshold definition for high-resolution scans (1.4 $\mu \mathrm{m})$ and (c) for low-resolution scans (16 $\mu \mathrm{m})$ 


\section{Results and discussion}

The fibre orientation distributions were calculated by ellipsometry for $1.4 \mu \mathrm{m}, 3.2 \mu \mathrm{m}$ and $3.2 \mu \mathrm{m}$ resampled images, where individual fibres could be identified. The $8 \mu \mathrm{m}$ and $16 \mu \mathrm{m}$ images were not analysed due to segmentation algorithm's inability to identify individual fibres in these resolutions. AVIZO was used to analyse $1.4 \mu \mathrm{m}$ images and $3.2 \mu \mathrm{m}$ resampled images, its use for other resolutions failed. Using the structure tensor analysis method, it was possible to analyse micro-CT images of $1.4 \mu \mathrm{m}, 3.2 \mu \mathrm{m}, 8 \mu \mathrm{m}$ and $16 \mu \mathrm{m}$ resolutions.

\subsection{Consistency of results for a given resolution and different locations}

Consistency of the fibre orientation distributions calculated by the ellipsometry method for different section plane locations is observed, for XY and ZX sections, for $1.4 \mu \mathrm{m}$ images (see Figure $4 \mathrm{a}$ and $\mathrm{b}$ ). The number of the analysed fibres (ellipses) in each plane ranged from 700 to 850. The distributions were compared using the Mann-Whitney U-test [39]. This test showed Pvalue higher than 0.05 for the distributions from different parallel planes of one specimen, both for $\mathrm{XY}$ and ZX sections. Therefore, there is not enough evidence to reject the hypothesis of consistency of results, and it can be concluded that each presented histogram describes the same fibre orientation distribution of the whole material. This result allows merging of the samplings provided by the three planes to obtain the more accurate description of the fibre orientations of the whole volume.

Figure 4 also presents ODFs obtained by the structure tensor analysis of different $1.4 \mu \mathrm{m}$ scan locations of one specimen (see Figure 4c) and for the same location, but with different discretization parameters (see Figure 4d). The U-test again supported the consistency of the results for different locations.
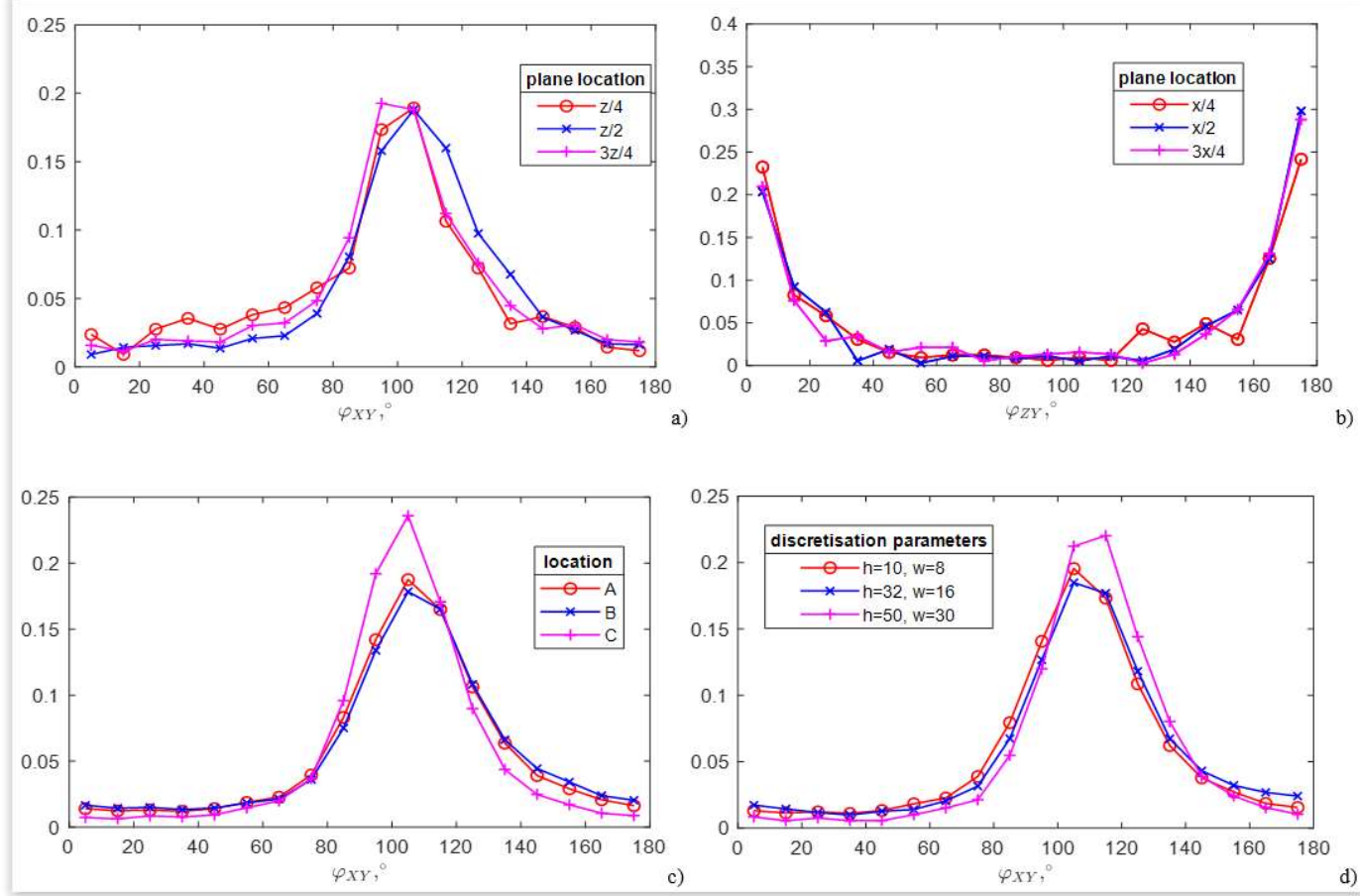

Figure 4: OFD ( $\varphi$-angles) obtained from 1.4-micron images: (a) by ellipsometry for different XY plane locations in one specimen ( $z$ being the VOI height), (b) by ellipsometry for different ZY plane locations in one specimen ( $x$ being the VOI length) (c) by structure tensor analysis for different locations, and (d) by structure tensor analysis for different discretization parameters. 
One can conclude that the ODF of the fibres in the studied sample does not depend on the image location or slice location within the image, and hence is a physical property of the sample. The samplings of the orientations obtained in different locations and different section planes can be merged to increase statistical representability of the reconstructed ODF.

Comparison of the histograms shown in Figure $4 \mathrm{~d}$ shows that the structure tensor-based orientation calculation is not sensitive to discretisation or averaging parameters when highresolution scans are used. However, when a very large window size is taken, the graph starts to show incorrect results $(\mathrm{h}=50$ pixels $=70 \mu \mathrm{m}, \mathrm{w}=30$ pixels $=42 \mu \mathrm{m}$ in Figure $4 \mathrm{~d})$. Therefore, for all subsequent calculations, the discretization parameters are selected in such a way that the window size is approximately equal to the fibre diameter.

\subsection{Resolution dependencies}

Figure 5 shows the difference in results of ellipsometry analysis of $1.4 \mu \mathrm{m}$ and $3.2 \mu \mathrm{m}$ images for a total number of analysed ellipses of about 2900. Results in XY and YZ planes for different resolutions repeat each other with small deviations. However, there is a difference in the ZX results: the $1.4 \mu \mathrm{m}$ results show the prevailing direction of the fibres about 90 degrees whereas the $3.2 \mu \mathrm{m}$ results show a noisy distribution but following on average the same trend. This difference is due to the low resolution of the $3.2 \mu \mathrm{m}$ scan: the fibre diameter corresponds to less than five pixels. In this case, the segmentation algorithm cannot identify fibres with sufficient accuracy and even a difference of just one pixel can change the ellipse angle significantly. The reason the problem appear in ZX plane orientation is probably the lower degree of fibre orientation in this plane. 

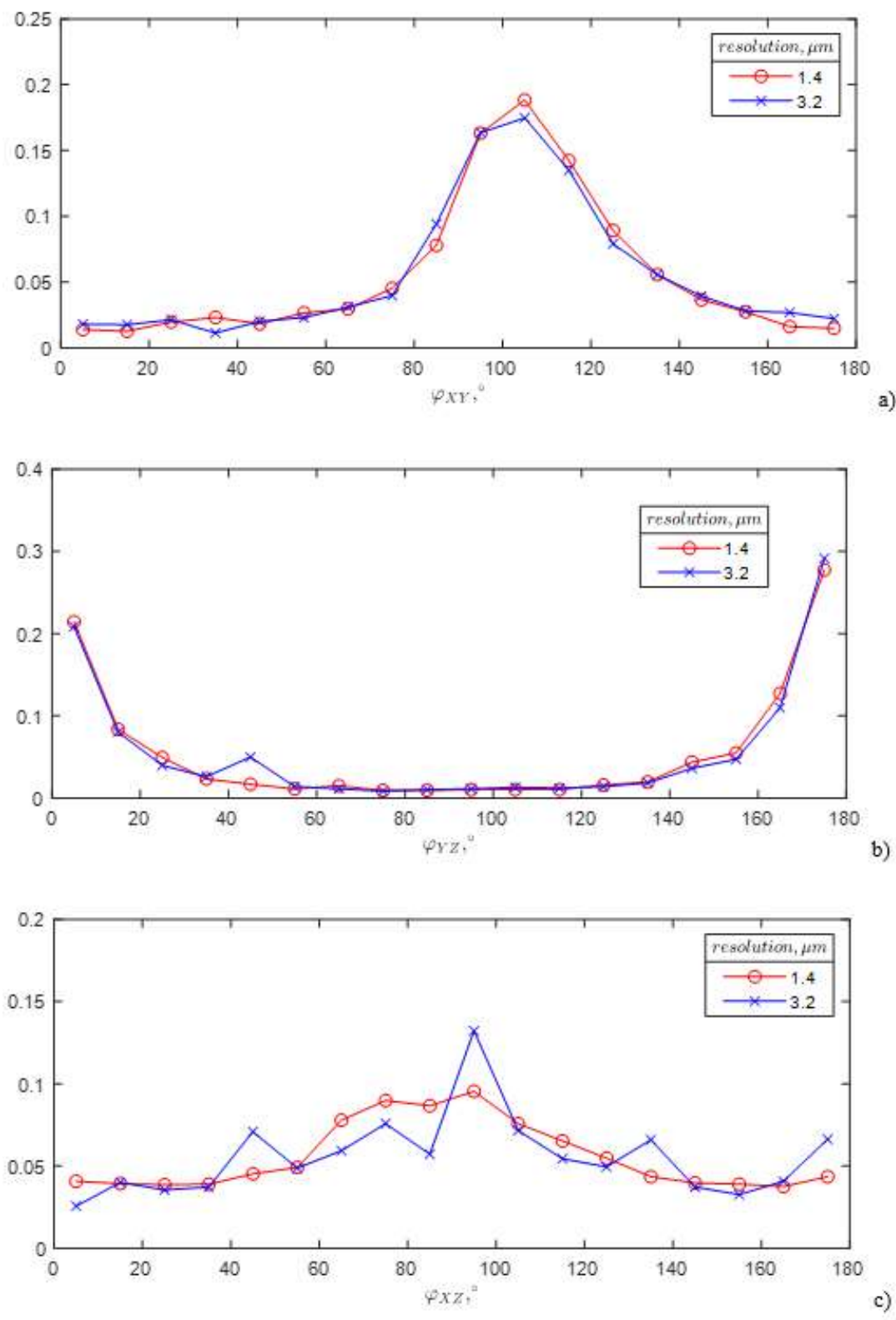

Figure 5: OFD ( $\varphi$-angles) obtained by ellipsometry for different resolutions: (a) XY plane, (b) YZ plane and (c) XZ plane.

The structure tensor analysis shows similar OFDs for different resolutions (see Figure 6). Sharper main orientations are observed on high-resolutions, however, all of them pass the U-test at a 0.05 level. This observation is explained by the use of large integration windows that average primary orientation when low-resolution images are analysed.

In addition, comparison between real high-resolution and synthetically generated low-resolution images was done. It helps to understand how the high-fidelity methods are sensitive to image quality [20]. There is a strong correlation of results calculated from original $1.4 \mu \mathrm{m}$ image and $3.2 \mu \mathrm{m}$ downscaled images when both ellipsometry and Avizo are used (Figure 7). When images were downscaled more than $3.2 \mu \mathrm{m} /$ pixel resolution, ellipsometry and Avizo fail to recognise individual fibres correctly. Therefore, if these methods can identify individual objects, they provide adequate results of fibre orientation distributions. 

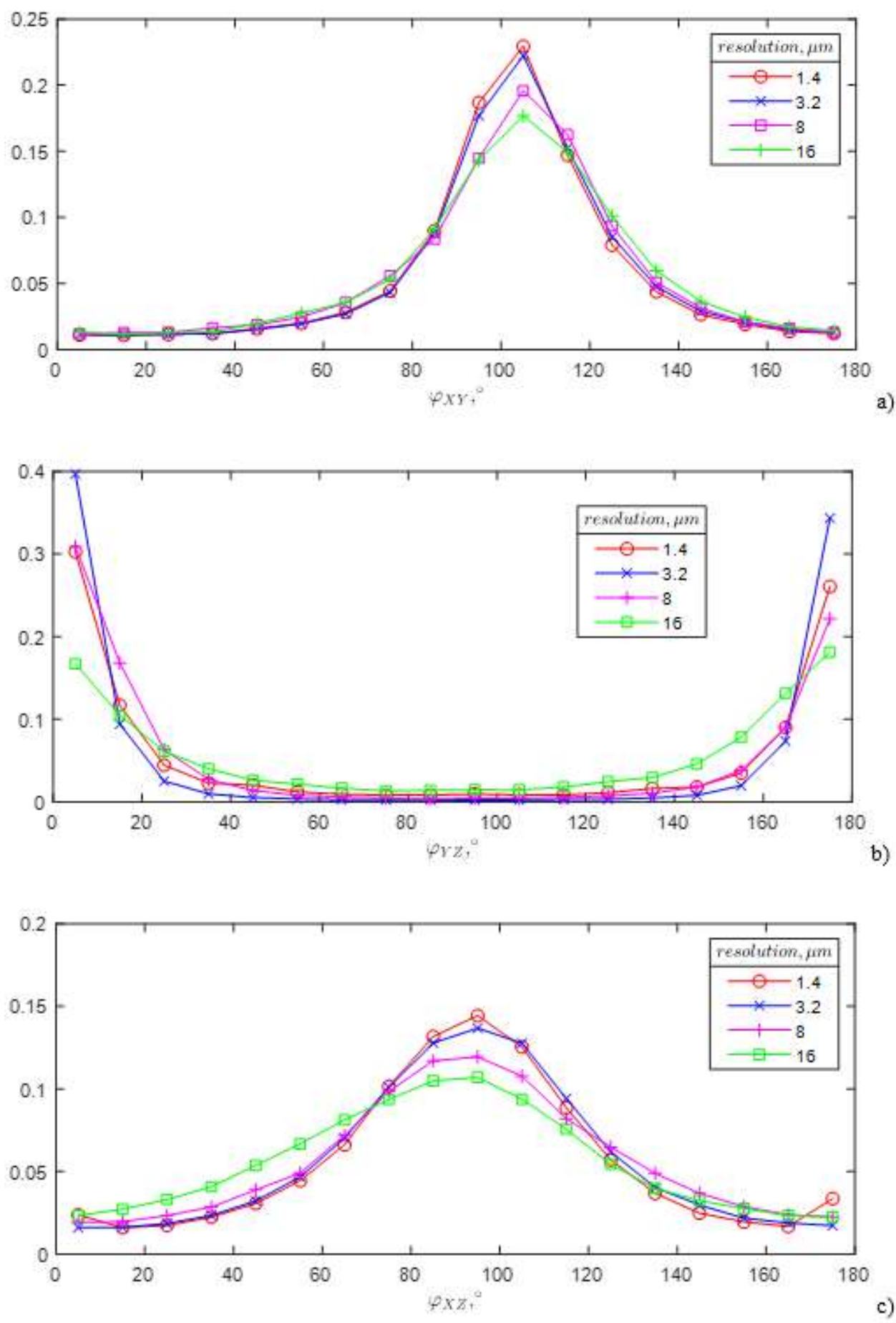

Figure 6: OFD ( $\varphi$-angles) obtained by structure tensor analysis for different resolutions: (a) XY plane, (b) YZ plane and (c) XZ plane. 


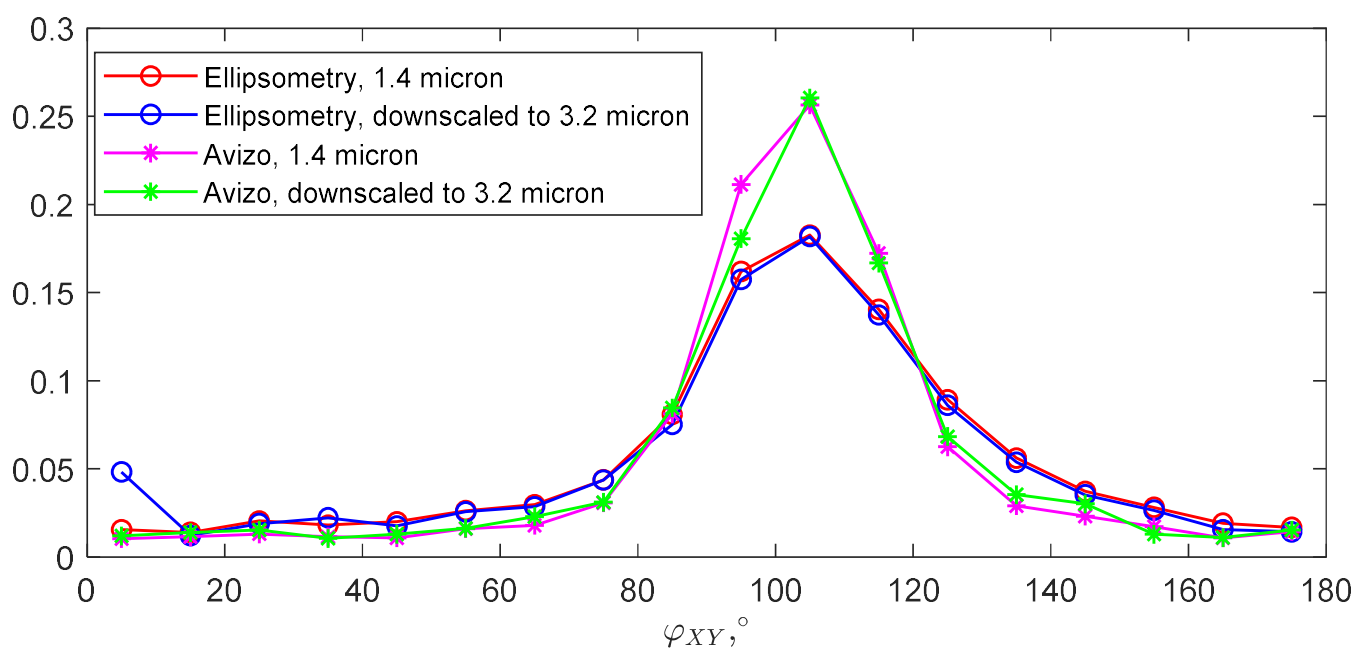

Figure 7: Comparison of OFDs ( $\varphi$-angles on XY plane) for original $1.4 \mu m$ images and downscaled 3.2 $\mu \mathrm{m}$ using ellipsometry and Avizo

\subsection{Comparison of the methods}

The ellipsometry analysis of the $1.4 \mu \mathrm{m}$ images was used as the reference in the comparison of the high-fidelity analysis with the structure tensor method. Ellipsometry analysis of $1.4 \mu \mathrm{m}$ images was compared with Avizo fibre reconstruction for the $1.4 \mu \mathrm{m}$ images and with $1.4 \mu \mathrm{m}$ and $16 \mu \mathrm{m}$ images as the best and the worst case for the structure tensor analysis. It can be seen that the obtained OFDs are similar (see Figure 8). However, the results should be subjected to MannWhitney U-tests to obtain a quantitative description of the distributions. Table 1 summarises the P-values obtained by the Mann-Whitney U-tests as well as the mean values and standard deviations of the distributions. The P-values are much higher than 0.05 , and the mean and standard deviations are similar for all three cases. This confirms the good correlations between the distributions obtained by ellipsometry, AVIZO and structure tensor analysis.

Table 1: Results of Mann-Whitney U-test (compared to ellipsometry), mean and standard deviation values of $\varphi$-angles.

\begin{tabular}{|c|c|c|c|c|}
\hline & & $\mathrm{XY}$ & ZY & $\mathrm{ZX}$ \\
\hline \multirow{2}{*}{$\begin{array}{l}\text { Mann-Whitney U- } \\
\text { test P-value }\end{array}$} & $\begin{array}{l}\text { Structure tensor } \\
\qquad(16 \mu \mathrm{m})\end{array}$ & 0.32 & 0.13 & 0.73 \\
\hline & Avizo $(1.4 \mu \mathrm{m})$ & 0.76 & 0.38 & 0.79 \\
\hline \multirow{4}{*}{$\begin{array}{c}\text { Mean value of } \\
\varphi \text {-angles, degrees }\end{array}$} & $\begin{array}{c}\text { Ellipsometry } \\
(1.4 \mu \mathrm{m})\end{array}$ & $101 \pm 32$ & $175 \pm 24$ & $90 \pm 45$ \\
\hline & $\begin{array}{l}\text { Structure tensor } \\
\qquad(16 \mu \mathrm{m})\end{array}$ & $102 \pm 31$ & $172 \pm 27$ & $88 \pm 39$ \\
\hline & $\begin{array}{l}\text { Structure tensor } \\
(1.4 \mu \mathrm{m})\end{array}$ & $104 \pm 31$ & $178 \pm 26$ & $89 \pm 36$ \\
\hline & Avizo $(1.4 \mu \mathrm{m})$ & $101 \pm 29$ & $177 \pm 17$ & $89 \pm 32$ \\
\hline
\end{tabular}




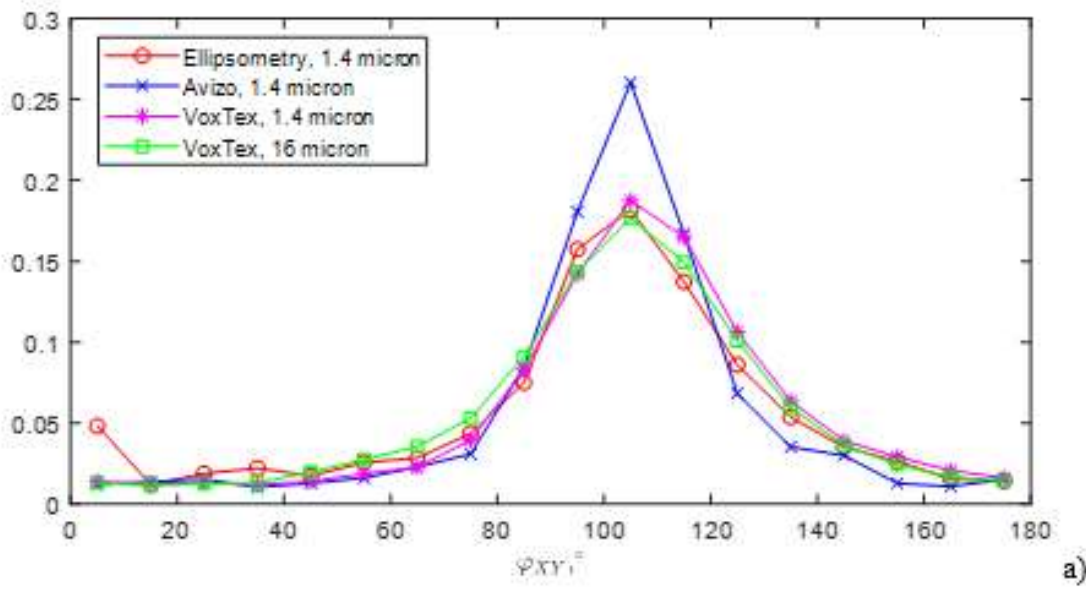

a)
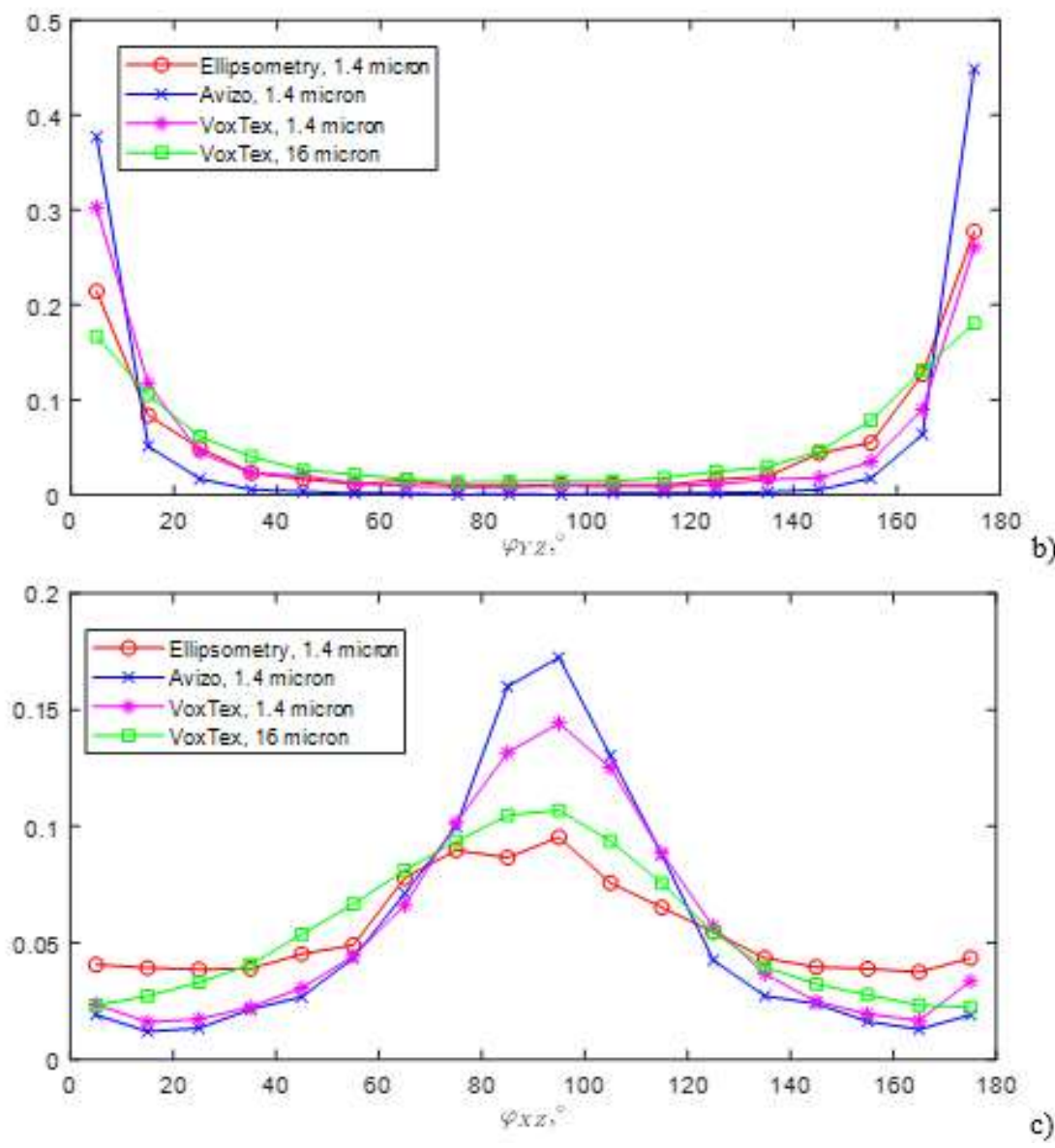

Figure 8: Comparison of OFDs ( $\varphi$-angles) calculated using structure tensor analysis (VoxTex), ellipsometry and Avizo software. The cumulative data for all locations (VoxTex) and section planes (ellipsometry): (a) $X Y$, (b) $Y Z$, (c) $X Z$.

Table 2 compares the orientation tensors calculated using the three different methods. The tensors show a correlation with tensor difference (see equation (6)) of 0.05 for structure tensor analysis and 0.08 for Avizo software of trace components and 0.15 for all components of the orientation tensors. In addition, the degrees of orientation and the cosine similarities are calculated using equations (4) and (5). All three methods show the same main fibre direction, with Avizo 
describing a more pronounced main direction, and ellipsometry a less pronounced one (see Figure $8 \mathrm{a}, \mathrm{c})$.

Table 2: Orientation tensors.

\begin{tabular}{|c|c|c|c|c|c|c|c|c|c|c|c|c|c|}
\hline \multicolumn{2}{|c|}{$\begin{array}{l}\text { Analysis } \\
\text { method }\end{array}$} & \multicolumn{3}{|c|}{ Ellipsometry $(1.4 \mu \mathrm{m})$} & \multicolumn{3}{|c|}{$\begin{array}{c}\text { Structure tensor (16 } \\
\mu \mathrm{m})\end{array}$} & \multicolumn{3}{|c|}{$\begin{array}{c}\text { Structure tensor (1.4 } \\
\mu \mathrm{m})\end{array}$} & \multicolumn{3}{|c|}{ Avizo $(1.4 \mu \mathrm{m})$} \\
\hline \multicolumn{2}{|c|}{$\begin{array}{l}\text { Orientation } \\
\text { tensor }\end{array}$} & {$\left[\begin{array}{c}0.182 \\
-0.145 \\
0.008\end{array}\right.$} & $\begin{array}{c}-0.145 \\
0.693 \\
-0.081\end{array}$ & $\left.\begin{array}{c}0.008 \\
-0.081 \\
0.125\end{array}\right]$ & {$\left[\begin{array}{c}0.194 \\
-0.139 \\
0.003\end{array}\right.$} & $\begin{array}{c}-0.139 \\
0.652 \\
0.02\end{array}$ & $\left.\begin{array}{c}0.003 \\
0.02 \\
0.154\end{array}\right]$ & {$\left[\begin{array}{c}0.228 \\
-0.186 \\
0.001\end{array}\right.$} & $\begin{array}{c}-0.186 \\
0.699 \\
0.02\end{array}$ & $\begin{array}{l}0.001 \\
-0.01 \\
0.073\end{array}$ & {$\left[\begin{array}{c}0.2 \\
-0.151 \\
-0.006\end{array}\right.$} & $\begin{array}{c}-0.151 \\
0.739 \\
0.009\end{array}$ & $\left.\begin{array}{c}-0.006 \\
0.009 \\
0.061\end{array}\right]$ \\
\hline \multirow{2}{*}{ 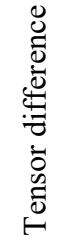 } & $\begin{array}{l}\text { trace } \\
\text { comp. }\end{array}$ & \multicolumn{3}{|c|}{-} & \multicolumn{3}{|c|}{0.05} & \multicolumn{3}{|c|}{0.05} & \multicolumn{3}{|c|}{0.08} \\
\hline & $\begin{array}{c}\text { all } \\
\text { comp. }\end{array}$ & \multicolumn{3}{|c|}{-} & \multicolumn{3}{|c|}{0.15} & \multicolumn{3}{|c|}{0.13} & \multicolumn{3}{|c|}{0.15} \\
\hline \multicolumn{2}{|c|}{$\begin{array}{l}\text { Degree of } \\
\text { Orientation }\end{array}$} & \multicolumn{3}{|c|}{0.56} & \multicolumn{3}{|c|}{0.61} & \multicolumn{3}{|c|}{0.63} & \multicolumn{3}{|c|}{0.67} \\
\hline \multicolumn{2}{|c|}{$\begin{array}{l}\text { Cosine } \\
\text { Similarity }\end{array}$} & \multicolumn{3}{|c|}{-} & \multicolumn{3}{|c|}{0.99} & \multicolumn{3}{|c|}{0.99} & \multicolumn{3}{|c|}{0.99} \\
\hline
\end{tabular}

\section{Conclusion}

3D micro-CT images of a random glass fibre composites were used to compare high-fidelity and structure tensor methods for fibre orientation. Micro-CT images of 1.4, 3.2, 8 and $16 \mu \mathrm{m}$ resolutions were used. The classical method of fibre cross-section analysis (ellipsometry) and 3D fibre identification (Avizo) were employed as high-fidelity methods. These two methods were able to handle only 1.4 (both Avizo and ellipsometry) and $3.2 \mu \mathrm{m}$ (ellipsometry only) images due to the inability to identify individual objects on low-resolution images. Structure tensor-based analysis was successful for all the resolutions. The orientation analyses of Avizo $(1.4 \mu \mathrm{m})$, ellipsometry $(1.4$ and $3.2 \mu \mathrm{m})$ and structure tensor method $(1.4,3.2,8$ and $16 \mu \mathrm{m})$ all generated orientation distributions that were statistically similar based on the Mann-Whitney U-tests for distributions and indices of tensor similarity for the orientation tensors. The structure tensor analysis was free from errors in theta-angle calculations compared to ellipsometry. The drawback of Avizo is that the technique of 3D fibre identification requires high computational resources.

The following conclusions can be drawn on the usability of the structure tensor analysis (VoxTex software), as demonstrated in the analysis of a typical sample of random glass fibre/PP composite:

1. For resolutions that allow individual fibre recognition, the structure tensor analysis of fibre orientation returns the results similar with high-fidelity methods, with a 5\% confidence level.

2. For image resolutions that do not allow individual fibre recognition, the structure tensor continues generating fibre orientation distributions and orientation tensors consistent with higher resolution images, up to the pixel size close to the fibre diameter.

\section{Acknowledgments}

The research visit of RK to KU Leuven has been funded by Skoltech. LM is an Early Stage Researcher in the Marie Skłodowska-Curie European Training Network FiBreMoD, funded by 
Horizon 2020 program of European Commission, research and innovation programme under the Marie Skłodowska-Curie grant agreement No 722626. The work is partially funded by SIM/VLAIO (Flanders) in T4G project running in NANOFORCE program and is supported by Toray Chair for Composite Materials@ KU Leuven, held by SL. The micro-CT images have been acquired on the X-ray computed tomography facilities at KU Leuven, maintained under the supervision of Prof. M. Wevers and financed by the Hercules Foundation and the KU Leuven Research Council (project C24/17/052). The authors are grateful for discussions with Dr. Ilya Straumit for the VoxTex calculations.

Data availability statement The data used in this research will be made available on a request.

\section{References}

1. K. Friedrich, A.A. Almajid, Manufacturing aspects of advanced polymer composites for automotive applications, Appl. Compos. Mater. 20 (2013) 107-128.

2. S.G. Advani, C.L. Tucker, The Use of Tensors to Describe and Predict Fiber Orientation in Short Fiber Composites, J. Rheol.. 31 (1987) 751-784.

3. R.S. Bay, C.L. Tucker, Fiber orientation in simple injection moldings. Part I: Theory and numerical methods, Polym. Compos. 13 (1992) 317-331.

4. C. Binetruy, F. Chinesta, R. Keunings, Flows in Polymers, Reinforced Polymers and Composites: A Multi-Scale Approach, Springer, 2015.

5. C. Eberhardt, A. Clarke, M. Vincent, T. Giroud, S. Flouret, Fibre-orientation measurements in short-glass-fibre compsites - II: A quantitative error estimate of the 2D image analysis technique, Compos. Sci. Technol. 61 (2001) 1961-1974.

6. K.S. Lee, S.W. Lee, J.R. Youn, T.J. Kang, K. Chung, Confocal Microscopy Measurement of the Fiber Orientation in Short Fiber Reinforced Plastics, Fibers Polym. 2 (2001) 41-50.

7. R. Blanc, C. Germain, J.P. Da, P. Baylou, M. Cataldi, Fiber orientation measurements in composite materials, Compos. Part A Appl. Sci. Manuf. 37 (2006) 197-206.

8. B. Mlekusch, Fibre orientation in short-fibre-reinforced thermoplastics II. Quantitative measurements by image analysis, Compos. Sci. Technol. 59 (1999)

9. I.M. Hine, P. J., Davidson, N., Duckett, R. A., \& Ward, Measuring the fibre orientation and modelling the elastic properties of injection-moulded long-glass-fibre-reinforced nylon, Compos. Sci. Technol. 53 (1995) 125-131.

10. R. Blanc, P. Westenberger. Comparison of fiber orientation analysis methods in Avizo. in: 7th Conference on Industrial Computed Tomography (iCT 2017), Leuven, Belgium, 2017 (http://www.ndt.net/?id=20865, accessed on 07.02.2019)

11. J.P. Suuronen, A. Kallonen, M. Eik, J. Puttonen, R. Serimaa, H. Herrmann, Analysis of short fibres orientation in steel fibre-reinforced concrete (SFRC) by X-ray tomography, J. Mater. Sci. 48 (2013) 1358-1367.

12. F.D.E. Pascalis, M. Nacucchi, Relationship between the anisotropy tensor calculated through global and object measurements in high-resolution X-ray tomography on cellular and composite materials, J. Microsc. 273 (2019) 65-80.

13. P.A. Hessman, T. Riedel, F. Welschinger, K. Hornberger, T. Böhlke, Microstructural analysis of short glass fiber reinforced thermoplastics based on x-ray micro-computed tomography, Compos. Sci. Technol. 183 (2019) 107752.

14. T. Wolfinger, P. Westenberger, Evaluation of specific network properties of paper using computer tomography and the latest Avizo Fire analysing tools, in: 5th Conference on Industrial Computed Tomography (iCT 2014), Wels, Austria, 2014 (http://www.ndt.net/?id=15690, accessed on 12.02.2019) 
15. P. Westenberger, P. Estrade, D. Lichau, Fibre Orientation Visualization with AvizoFire, in: 4th Conference on Industrial Computed Tomography (iCT 2012), Wels, Austria, 2014 (http://www.ndt.net/?id=13711, accessed on 12.02.2019)

16. W.J. Whitehouse, The quantitative morphology of anisotropic trabecular bone, J. Microsc. 101 (1974) 153-168.

17. T.P. Harrigan, R.W. Mann, Characterization of microstructural anisotropy in orthotropic materials using a second rank tensor, J. Mater. Sci. 19 (1984) 761-767.

18. S.C. Cowin, Wolff's Law of Trabecular Architecture at Remodeling Equilibrium, J. Biomech. Eng. 108 (1986) 83.

19. A. Odgaard, E.B. Jensen, H.J.G. Gundersen, Estimation of structural anisotropy based on volume orientation. A new concept, J. Microsc. 157 (1990) 149-162.

20. G. Bravais, P. Latil, A. Sonzogni, O. Guiraud, B. Fayard, Extraction of local fibre orientation in composite media from X-ray micro-tomography: a comparative study, in: 14th French InterPore Conference on Porous Media (FIPCM 2018), Nantes, France, 2018

21. I. Straumit, S. V. Lomov, M. Wevers, Quantification of the internal structure and automatic generation of voxel models of textile composites from X-ray computed tomography data, Compos. Part A Appl. Sci. Manuf. 69 (2015) 150-158.

22. I. Baran, I. Straumit, O. Shishkina and S.V. Lomov, X-ray computed tomography characterization of manufacturing induced defects in a glass/polyester pultruded profile, Comp. Struct. 195 (2018) 74-82.

23. N. Nguyen, M. Mehdikhani, I. Straumit, L. Gorbatikh, L. Lessard, S.V. Lomov, MicroCT measurement of fibre misalignment: application to carbon/epoxy laminates manufactured in autoclave and by vacuum assisted resin transfer moulding, Compos. Part A Appl. Sci. Manuf. 104 (2018) 14-23.

24. Y. Wan, I. Straumit, J. Takahashi, S.V. Lomov, Micro-CT analysis of internal geometry of chopped carbon fiber tapes reinforced thermoplastics, Compos. Part A Appl. Sci. Manuf. 91-1 (2016) 211-221.

25. Y. Liu, I. Straumit, D. Vasiukov, S.V. Lomov, S. Panier, Prediction of linear and nonlinear behavior of 3D woven composite using mesoscopic voxel models reconstructed from X-ray micro-tomography, Comp. Struct. 179 (2017) 568-579.

26. J. Tang, Y. Swolfs, M.L. Longana, H. Yu, M.R. Wisnom, S.V. Lomov, L. Gorbatikh, Hybrid composites of aligned discontinuous carbon fibers and self-reinforced polypropylene under tensile loading, Compos. Part A Appl. Sci. Manuf. 123 (2019) 97-107.

27. D. Alexander, J. Gee, and R. Bajcsy, Similarity measures for matching diffusion tensor images, Proceedings of the British Machine Vision Conference, 93-102, 1999.

28. J. Weissenböck, M. Arikan, D. Salaberger, J. Kastner, J.D. Beenhouwer, J. Sijbers, T. Raab-wernig, E. Gröller, C. Heinzl, Comparative Visualization of Orientation Tensors in FiberReinforced Polymers, in: 8th Conference on Industrial Computed Tomography (iCT 2018), Wels, Austria, 2018 (https://www.ndt.net/?id=21912, accessed on 14.04.2019)

29. Y.T. Zhu, W.R. Blumenthal, T.C. Lowe, Determination of Non-Symmetric 3-D FiberOrientation Distribution and Average Fiber Length in Short-Fiber Composites, J. Compos. Mater. 31 (1997) 1287-1301.

30. M. Jane, V. Andersen, K. Conradsen, L. Pilgaard, A. Bjorholm, Statistical validation of individual fi bre segmentation from tomograms and microscopy, Compos. Sci. Technol. 160 (2018) 208-215. 
31. B. Weber, G. Greenan, S. Prohaska, D. Baum, H. Hege, T. Müller-reichert, A.A. Hyman, J. Verbavatz, Automated tracing of microtubules in electron tomograms of plastic embedded samples of Caenorhabditis elegans embryos, J. Struct. Biol. 178 (2012) 129-138.

32. P. Westenberger, R. Blanc, Advanced Fiber Evaluation Workflows, in: 6th Conference on Industrial Computed Tomography (iCT 2016), Wels, Austria, 2016 (http://www.ndt.net/?id=18767, accessed on 17.02.2019)

33. ThermoScientific Avizo Software 9 User's Guide, 2018 (https://assets.thermofisher.com/TFS-Assets/MSD/Product-Guides/user-guide-Avizosoftware.pdf, accessed on 21.02.2019).

34. Z. Tabor, R. Petryniak, Z. Latała, T. Konopka, Medical Engineering \& Physics The potential of multi-slice computed tomography based quantification of the structural anisotropy of vertebral trabecular bone, Med. Eng. Phys. 35 (2013) 7-15.

35. M.D. Budde, J.A. Frank, NeuroImage Examining brain microstructure using structure tensor analysis of histological sections, Neuroimage. 63 (2012) 1-10.

36. M. Krause, J. M. Hausherr, B. Burgeth, C. Herrmann, W. Krenkel, Determination of the fibre orientation in composites using the structure tensor and local X-ray transform, J Mater Sci 45 (2010) 888-896.

37. P. Pinter, S. Dietrich, B. Bertram, L. Kehrer, P. Elsner, K.A. Weidenmann, Comparison and error estimation of 3D fibre orientation analysis of computed tomography image data for fibre reinforced composites, NDT and E International 95 (2018) 26-35.

38. I. Arganda-Carreras, V. Kaynig, C. Rueden, K.W. Eliceiri, J. Schindelin, A. Cardona, H.S. Seung, Trainable Weka Segmentation: a machine learning tool for microscopy pixel classification, Bioinformatics. 33 (2018) 2424-2426.

39. F.S. Nahm, Nonparametric statistical tests for the continuous data: The basic concept and the practical use, Korean J. Anesthesiol. 69 (2016) 8-14. 